\title{
SÍNTESIS Y CARACTERIZACIÓN DE NUEVOS COPOLÍMEROS DE INJERTO TERMOSENSIBLES A PARTIR DE N-ISOPROPILACRILAMIDA Y MACROMONÓMEROS DE 2-METIL-2-OXAZOLINA
}

\author{
Ana María Paola Palacios ${ }^{\mathrm{a}}$, Juan Carlos Rueda ${ }^{\mathrm{a}^{*}}$
}

\begin{abstract}
RESUMEN
Fueron sintetizados nuevos copolímeros injertados a partir de la polimerización por radicales libres de N-isopropilacrilamida (NIPAAm) y macromonómeros de 2-metil-2-oxazolina (MM) iniciada por el 2,2'-azobisisobutironitrilo en dimetilformamida. Los macromonómeros, de grados de polimerización 31 y 62, fueron sintetizados mediante la polimerización catiónica por apertura de anillo de 2-metil-2-oxazolina iniciada por el clorometilestireno en presencia de ioduro de sodio. Los macromonómeros y copolímeros injertados fueron caracterizados por espectrometría de resonancia magnética nuclear de protones $\left({ }^{1} \mathrm{H}-\mathrm{RMN}\right)$ y la temperatura de transición conformacional fue determinada mediante mediciones turbidimétricas y espectroscopía ${ }^{1} \mathrm{H}-\mathrm{RMN}$.

La razón molar NIPAAm/MM en el copolímero, determinada por ${ }^{1} \mathrm{H}-\mathrm{RMN}$, fue mayor que en la alimentación al reactor debido a la mayor reactividad del NIPAAm por su mayor difusividad en el medio reaccionante debido a su menor peso molecular. La temperatura de transición conformacional (LCST) de los copolímeros injertados estuvo en el rango de 33 a $38{ }^{\circ} \mathrm{C}$ y la intensidad de la misma fue función de la razón molar de NIPAAm/ macromonómero dentro del copolímero injertado. Se incrementó el valor de la LCST, y al mismo tiempo disminuyó su intensidad, al disminuir la razón molar NIPAAm/MM en el copolímero. La LCST también disminuyó aún más en intensidad al aumentar el grado de polimerización del macromonómero. A partir de la temperatura LCST, los copolímeros injertados con un relativamente alto contenido de MM podrían formar micelas o agregados moleculares tipo "core-shell” en solución acuosa, que estarían formados por un núcleo con segmentos colapsados de poliNIPAAm y una corona formada de cadenas hidrofílicas de polimetiloxazolina, las cuales impiden parcialmente un colapso intermolecular de estos agregados.
\end{abstract}

Palabras clave: Copolímero de injerto, NIPAAm, termosensibilidad, macromonómero, polioxazolinas.

\footnotetext{
a Laboratorio de Polímeros, Sección Física, DGI, Pontificia Universidad Católica del Perú, Av. Universitaria 1801, San Miguel, Lima, Perú, jrueda@pucp.edu.pe
} 


\title{
SYNTHESIS AND CHARACTERIZATION OF NEW THERMOSENSITIVE GRAFT COPOLYMERS FROM N-ISOPROPYLACRYLAMIDE AND 2-METHYL-2-OXAZOLINE MACROMONOMERS
}

\begin{abstract}
New graft copolymers were synthesized from the free radical polymerization of $\mathrm{N}$-isopropylacrylamide (NIPAAm) and 2-methyl-2-oxazoline (MM) macromonomers initiated by 2,2'-azobisisobutyronitrile in dimethylformamide. The macromonomers, with polymerization degrees 31 and 62, were synthesized by ring-opening cationic polymerization of 2-methyl-2-oxazoline initiated by chloromethylstyrene in the presence of sodium iodide. The grafted macromonomers and copolymers were characterized by proton nuclear magnetic resonance spectrometry $\left({ }^{1} \mathrm{H}-\mathrm{RMN}\right)$ and the conformational transition temperature was determined by turbidimetric measurements and ${ }^{1} \mathrm{H}-\mathrm{RMN}$ spectroscopy. The NIPAAm / MM molar ratio in the copolymer, determined by ${ }^{1} \mathrm{H}-\mathrm{RMN}$, was higher than in the feed to the reactor due to the higher reactivity of the NIPAAm due to its greater diffusivity in the reacting medium due to its lower molecular weight. The conformational transition temperature (LCST) of the graft copolymers ranged from 33 to $38^{\circ} \mathrm{C}$ and the intensity of the same was a function of the molar ratio of NIPAAm / macromonomer within the graft copolymer. The value of the LCST increased, and at the same time its intensity decreased, as the NIPAAm / MM molar ratio decreased in the copolymer. The LCST also decreased even more in intensity as the degree of polymerization of the macromonomer increased. From the LCST temperature, the graft copolymers with a relatively high content of MM could form micelles or molecular aggregates type "core-shell" in aqueous solution, which would be formed by a core with collapsed segments of polyNIPAAm and a shell formed of hydrophilic polymethyloxazoline chains, which partially prevent an intermolecular collapse of these aggregates.
\end{abstract}

Key words: Graft copolymers, NIPAAm, thermoresponsive, macromonomer, polyoxazolines.

\section{INTRODUCCIÓN}

El campo de los "smart" polímeros o polímeros inteligentes ha despertado un gran interés tecnológico debido a la variedad de aplicaciones en las que se pueden utilizar estos materiales ya que son capaces de responder de forma reversible a estímulos externos como, por ejemplo, temperatura, $\mathrm{pH}$, luz, campo magnético y eléctrico.$^{1,2}$. Las principales aplicaciones de estos polímeros se encuentran en campos como liberación controlada de medicamentos, inmovilización de proteínas y enzimas, sensores y actuadores, óptica, entre otros ${ }^{2,3}$.

Uno de los monómeros más utilizados para fabricar polímeros inteligentes ("smart polymers") sensibles a la temperatura es la $\mathrm{N}$-isopropilacrilamida (NIPAAm) $)^{4,5}$. Cuando el poliNIPAAm se disuelve en agua, tiene una temperatura de transición conformacional (Lower Critical 
Solution Temperature - LCST) de $32{ }^{\circ} \mathrm{C}$, que debido a su cercanía a la temperatura del cuerpo humano, podría utilizarse para elaborar biomateriales inteligentes ${ }^{3,4}$. A temperaturas superiores al LCST, los segmentos poliméricos de poliNIPAAm pasan de ser hidrofílicos a hidrofóbicos y entonces comienzan a interactuar entre sí de una manera más intensa que con el agua y esto lleva a la precipitación o dispersión del polímero en el medio acuoso ${ }^{4}$. La LCST del poliNIPAAM puede disminuir o aumentar, cuando el NIPAAm es copolimerizado con comonómeros hidrofóbicos o hidrofílicos, respectivamente ${ }^{5,7}$. La LCST también se puede incrementar adicionando a la solución acuosa de poliNIPAAm sales inorgánicas o surfactantes. Las propiedades de estos polímeros y su aplicabilidad están determinadas, también, por las funciones químicas del comonómero del NIPAAm y el peso molecular del polímero ${ }^{7}$.

La síntesis de copolímeros estadísticos a partir de NIPAAm y acrilato de sodio produce un aumento del LCST del poliNIPAAm debido a la fuerte polaridad del acrilato de sodio y, adicionalmente, se obtiene sensibilidad al $\mathrm{pH}$ debido a la función química carboxilato de este monómero ${ }^{6,7}$. Sin embargo, una distribución aleatoria de unidades del acrilato de sodio dentro de la cadena de poliNIPAAm no permite obtener una fuerte intensidad de la respuesta térmica ni mantener la temperatura de transición LCST cercana a la temperatura del cuerpo humano ${ }^{7}$. Una posible solución a este problema podría ser que los grupos ácidos no estén en la cadena principal del PoliNIPAAm sino en cadenas laterales y esto se podría lograr copolimerizando el NIPAAm con macromonómeros que contengan grupos ácidos ${ }^{8,9}$. Los macromonómeros de poli(2-oxazolina), conteniendo grupos ácidos, podrían ser adecuados para lograr esta separación de fases y también pueden darles nuevas propiedades a los copolímeros injertados porque los grupos ácido pueden ser modificados posteriormente a diversas funciones químicas tales como aminas, tioles ${ }^{9,10}$.

La síntesis de poli(2-oxazolina) mediante polimerización catiónica de apertura de anillo ocurre en una forma "viva", o sea sin reacciones de interferencia (reacciones de terminación o de transferencia de cadena), por lo que el peso molecular y la funcionalidad de estos polímeros pueden controlarse con gran eficiencia permitiendo, por ejemplo, la introducción de grupos vinilo al inicio o al final de la cadena polimérica ${ }^{8-13}$. Se ha determinado que en la polimerización de este tipo de monómeros ocurre una transferencia de cadena solo para razones molares iniciales de monómero/iniciador mayores a $350^{12-14}$.

Anteriormente, nuestro grupo de investigación sintetizó copolímeros injertados de NIPAAm y 2-oxazolinas mediante el método "grafting from", y se obtuvieron copolímeros con un valor relativamente alto del grado de polimerización de las cadenas laterales ${ }^{15,16,18}$.

En este trabajo se presenta la síntesis, mediante el método del macromonómero, de copolímeros injertados termosensibles a partir de PNIPAAm y macromonómeros de 2-metil2-oxazolina. 


\section{PARTE EXPERIMENTAL}

\section{Materiales y equipos}

Se purificó N-isopropilacrilamida (NIPAAm) (Aldrich) mediante su recristalización en etanol absoluto y se secó al vacío. Se sintetizó 2-metil-2-oxazolina (MeOxa) mediante el método descrito en la literatura ${ }^{17}$ y se secó mediante destilación sobre hidruro de calcio. El clorometilestireno (CMS) (Aldrich, mezcla de isómeros meta (70 \% molar) y para (30 $\%$ molar) se purificó mediante destilación fraccionada antes de su uso. Se recristalizó 2,2'-azobis (isobutironitrilo) (AIBN) en metanol. El éter dietílico, el cloroformo y el metanol se destilaron antes de su uso. Se utilizó acetonitrilo anhidro (Merck), yoduro de sodio y nitrógeno, todos de alta pureza y fueron usados tal como recibidos. El poliNIPAAm (Aldrich) de peso molecular 20000 (Aldrich) se usó como fue recibido. El agua utilizada para todas las mediciones fue purificada y desionizada en un sistema de purificación Millipore Milli-Q. Todos los demás solventes y productos químicos fueron usados tal como fueron recibidos.

Las mediciones de resonancia magnética nuclear (RMN) fueron llevadas a cabo en un equipo Bruker Advance III 500 Spectrometer, operando a 500,13 MHz para $1 \mathrm{H}$. Como solventes se utilizaron agua y metanol deuterados y como estándar interno se usó el tetrametilsilano (TMS).

Las mediciones de turbidimetría fueron realizadas en el equipo UV/Vis Thermo Fisher Scientific Helios Gamma. El baño termotastizador Fischerbrand Modelo FBH-604 fue usado para variar la temperatura de la solución polimérica en los análisis turbidimétricos.

\section{Procedimiento experimental}

Síntesis del macromonómero de 2-metil-2-oxazolina (MM)

Los macromonómeros (MM) se sintetizaron mediante la copolimerización catiónica por apertura de anillo de 2-metil-2-oxazolina (MeOXA) iniciada por el clorometilestireno (CMS) en presencia de yoduro de sodio de acuerdo con el método descrito en la literatura ${ }^{9-11}$.

Procedimiento (MM-1): En un reactor de $100 \mathrm{~mL}$, se mezclaron, bajo corriente de nitrógeno seco, 7,19 g (84,4 mmol) de MeOXA, 0,84 g (5,6 mmol) de NaI y 0,43 g (2,81 $\mathrm{mmol})$ de CMS en $25 \mathrm{ml}$ de acetonitrilo anhidro. Esta mezcla se calentó a $78{ }^{\circ} \mathrm{C}$, bajo atmósfera de nitrógeno, durante 7 horas. Al final de este periodo se enfrió el sistema y se agregó una solución de $0,3 \mathrm{~g}$ de hidróxido de potasio en $2 \mathrm{~mL}$ de metanol. Posteriormente, la solución fue filtrada para eliminar las sales y se obtuvo el polímero mediante precipitación en éter dietílico. Se obtuvo 7,34 g de macromonómero MM-1 con un rendimiento del 97 \%. El MM-2 fue sintetizado en forma similar a MM-1 y se usó 2,11 mmol de CMS y 137,2 mmol de MeOXA en el caso de MM-2.

${ }^{1} \mathrm{H}$ RMN (CD $\left.{ }^{3} \mathrm{OD}\right) \delta(\mathrm{ppm}): 2,0-2,2\left(\mathrm{NC}(\mathrm{O}) \mathrm{CH}_{3}\right), 3,4-3,8\left(\mathrm{NCH}_{2} \mathrm{CH}_{2}\right), 5,2-5,3$ y 5,7-5,9 $\left(=\mathrm{CH}_{2}\right), 6,7-6,9$ (=CH-), 7,1-7,5 $\left(\mathrm{H}_{\text {arom }}\right)$. 
Mediante el análisis cuantitativo del espectro de ${ }^{1} \mathrm{H}-\mathrm{RMN}$, se determinó que los grados de polimerización (DP) de MM-1 y MM-2 fueron 31 y 62, respectivamente. Así, los pesos moleculares de los macromonómeros MM-1 y MM-2, determinados mediante RMN, fueron 2770 y $5410 \mathrm{~g} / \mathrm{mol}$, respectivamente.

\section{Sintesis de copolímeros injertados (CI)}

Procedimiento (CI-2): En un balón de $100 \mathrm{ml}$ y con entrada lateral de nitrógeno seco, fueron disueltos, en $8 \mathrm{ml}$ de dimetilformamida (DMF), 1,03 g de MM-1 (0,37 mmol), 1,26 g de NIPAAm (11,16 mmol) y 0,048 g de AIBN (0,29 mmol). Se burbujeó nitrógeno durante 10 minutos en la mezcla de reacción. El sistema fue cerrado y se calentó a $70{ }^{\circ} \mathrm{C}$ durante 24 horas, bajo agitación constante. Al término de este periodo, el sistema fue enfriado y adicionado al sistema de reacción $20 \mathrm{ml}$ de una mezcla de dioxano y cloroformo (v:v 50/50). Luego el polímero fue obtenido mediante precipitación en $400 \mathrm{~mL}$ de éter dietílico. Finalmente, el polímero fue secado y guardado en un desecador. Se obtuvo 1,90 g de polímero injertado (rendimiento: $82 \%$ ). Los demás copolímeros injertados se sintetizaron en forma similar.

El polímero fue caracterizado por ${ }^{1} \mathrm{H}-\mathrm{RMN}$ para confirmar su estructura y obtener la relación molar de NIPAAm/MM en el mismo.

${ }^{1} \mathrm{H}-\mathrm{RMN}\left(\mathrm{CD}_{3} \mathrm{OD}\right) \delta$ (ppm): 1,15 (CH3, NIPAAm); 1,3-1,9 (CH2CH, NIPAAm, MM-1); 2,0-2,2 ( $\left.\left.\mathrm{CH}_{3}, \mathrm{MM}-1\right) ; 3,4-3,7\left(\mathrm{NCH}_{2} \mathrm{CH}_{2}, \mathrm{MM}-1\right), 4,0\left(\mathrm{CH}_{\left(\mathrm{CH}_{3}\right.}\right)_{2}\right), 6,8-7,3(\mathrm{NH}) ; 7,5-8,2$ $\left(\mathrm{H}_{\mathrm{ar}}\right)$.

Determinación de la temperatura de transición conformacional (LCST) de los copolímeros injertados

La determinación de la temperatura de transición conformacional (LCST) de los copolímeros injertados se realizó mediante el método turbidimétrico.

Procedimiento: se pesaron $0,040 \mathrm{~g}$. de polímero injertado y se disolvieron en 4,0 $\mathrm{ml}$ de agua destilada $(\mathrm{pH}=5,7)$, de modo que la concentración en peso del polímero fuese $1 \%$. Luego, se filtró la solución polimérica y se colocó en una cubeta de un espectrómetro UV/Vis. También, se colocó una termocupla flexible dentro de la cubeta, de tal manera que se pudiera medir en todo momento la temperatura de la solución polimérica contenida en la misma. Se calentó gradualmente la solución acuosa y se procedió a medir con el espectrómetro UV/ Vis el porcentaje de transmitancia de la solución acuosa versus la temperatura de la misma a una longitud de onda de $500 \mathrm{~nm}$. A partir de un cierto valor de temperatura el valor de transmitancia comenzó a disminuir debido a la precipitación o dispersión del polímero en la fase acuosa. Los valores del porcentaje de transmitancia fueron graficados en función de la temperatura y se obtuvo una curva. Se tomó como el valor de la temperatura de transición (LCST) el punto de inflexión de la curva ${ }^{4,7}$. 


\section{RESULTADOS Y DISCUSIÓN}

Síntesis de los macromonómeros de poli (2-oxazolina) (MM)

Los macromonómeros (MM) se sintetizaron mediante la polimerización catiónica por apertura de anillo (PCAA) de 2-metil-2-oxazolina (MeOxa) iniciada por el clorometilestireno (CMS) en acetonitrilo (esquema 1). Se añadió yoduro de sodio al sistema de polimerización para coiniciar la reacción mediante el intercambio de halógeno. El grupo vinilo fue introducido por el iniciador CMS (método del "iniciador") ${ }^{9}$. Para evitar la polimerización prematura del grupo vinilo, se utilizó una concentración relativamente baja de CMS $(0,136 \mathrm{M})$, una baja temperatura de reacción de $78^{\circ} \mathrm{C}$ y un corto tiempo de reacción de $7 \mathrm{~h}^{9-11}$. La polimerización se terminó con una solución de $\mathrm{KOH}$ en metanol introduciendo así un grupo $\mathrm{OH}$ en el final de cadena. Los rendimientos de las reacciones de síntesis de los macromonómeros fueron mayores al $94 \%$. Los detalles experimentales y resultados obtenidos se resumen en la tabla 1.

La polimerización PCAA de las 2-oxazolinas ocurre vía polimerización "viva", esto quiere decir que solo ocurren las reacciones de iniciación y propagación sin que ocurran reacciones de interferencia (reacción de terminación o de transferencia de cadena). Por esta razón, es posible sintetizar los macromonómeros MM-1 y MM-2 con grados de polimerización predeterminados (tabla 1).
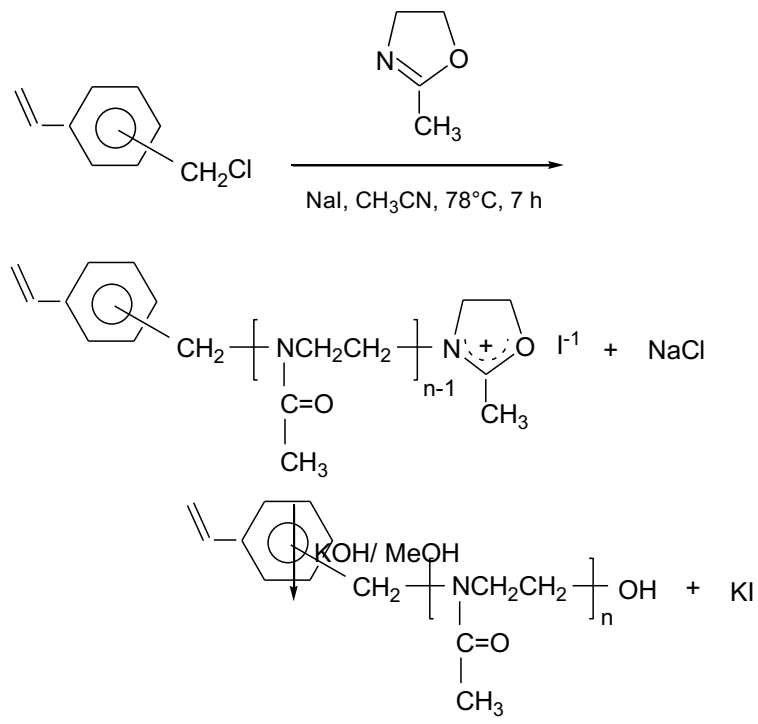

Macromonómero (MM)

Esquema 1. Síntesis del macromonómero de 2-metil-2-oxazolina. 
Tabla 1. Síntesis de los macromonómeros. Condiciones de reacción y resultados.

\begin{tabular}{lcccccc}
\hline MM $^{\mathrm{a}}$ & $\mathrm{CMS}^{\mathrm{b}}$ & $\begin{array}{c}\mathrm{MeOxa}^{\mathrm{c}} \\
(\mathrm{mmol})\end{array}$ & $\begin{array}{c}\mathrm{Nal}^{\mathrm{d}} \\
(\mathrm{mmol})\end{array}$ & $\begin{array}{c}\mathrm{MeOX} / \mathrm{CMS}^{\mathrm{e}} \\
(\mathrm{mmol})\end{array}$ & $\mathrm{MeOXA} \mathrm{CMS}^{\mathrm{f}}$ & $\begin{array}{c}\mathrm{R}^{\mathrm{g}} \\
(\%)\end{array}$ \\
\hline $\mathrm{MM}-1$ & 2,81 & 84,44 & 5,63 & 30 & 31 & 97 \\
$\mathrm{MM}-2$ & 2,11 & 137,20 & 4,22 & 65 & 62 & 95 \\
\hline
\end{tabular}

a) Denominación del macromonómero, b) Clorometilestireno, ${ }^{\text {c) }}$ 2-Metil-2-oxazolina, d) Ioduro de sodio, ${ }^{\text {e) }}$ Relación molar inicial de metiloxazolina y clorometilestireno en la alimentación al reactor f) Relacion molar de metiloxazolina y clorometilestireno en el macromonómero determinada mediante el espectro ${ }^{1} \mathrm{H}-\mathrm{RMN},{ }^{\mathrm{g})}$ Rendimiento de la polimerizacion, que es igual a la relación entre el peso de macromonómero obtenido versus la suma del peso inicial de iniciador (CMS) y monómero (MeOxa).

Los macromonómeros fueron solubles en agua, metanol y cloroformo. La estructura molecular de los macromonómeros (MM) fue confirmada mediante espectroscopía ${ }^{1} \mathrm{H}$ - RMN (ej. MM-1 en figura 1).

El grado de polimerización de los macromonómeros se determinó a partir de la comparación entre la integral de la señal del grupo metilo "f” de MeOxa $(2,1 \mathrm{ppm})$ versus las señales “a, á, b y c" del grupo de partida estirilo (figura 1). Los grados de polimerización de MM-1 y MM-2, así determinados por RMN, fueron de $31\left(\mathrm{Mn}_{\mathrm{RMN}}=2770\right)$ y $62\left(\mathrm{Mn}_{\mathrm{RMN}}=5410\right)$ para MM-1 y MM-2, respectivamente. Estos valores están en excelente concordancia con los valores de la razón molar inicial macromonómero/iniciador de 30 y 65 para MM-1 y MM-2, respectivamente (tabla 1). Este resultado evidenció que ocurrió una polimerización de tipo "viva" de la MeOXA.

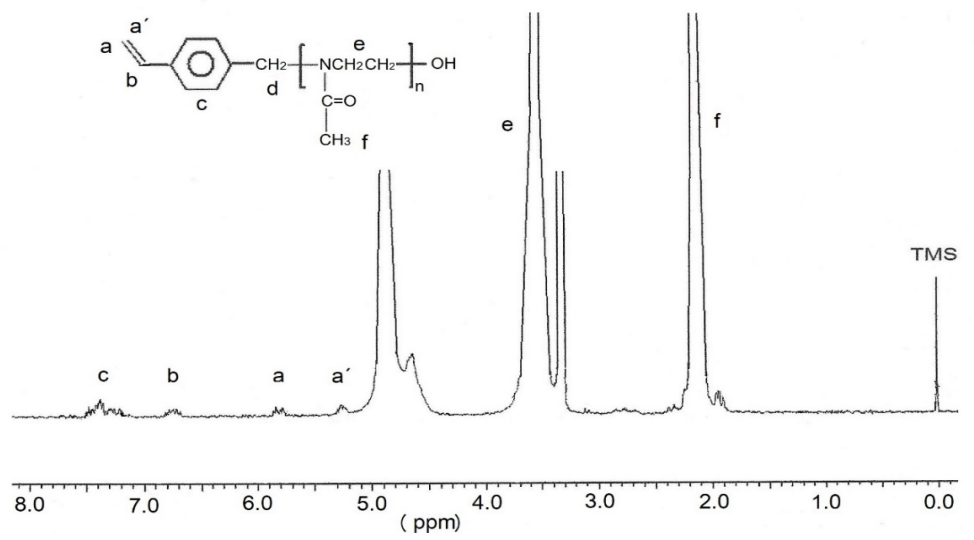

Figura 1. Espectro ${ }^{1} \mathrm{H}-\mathrm{RMN}$ del macromonómero $\mathrm{MM}-1$ en $\mathrm{CD}_{3} \mathrm{OD}$ a $25^{\circ} \mathrm{C}$. Las señales del espectro han sido asignadas a la estructura del polímero. Las señales en 3,3 y 4,9 ppm corresponden a las señales del metanol deuterado 
El mecanismo de polimerización puede ser descrito de la siguiente manera ${ }^{9}$ : En la reacción de iniciación ocurre un intercambio entre el átomo de cloro del clorometilestireno y el átomo de yodo del ioduro de sodio y de esta manera se genera el grupo funcional yoduro de bencilo, el cual es un grupo más eficiente que el cloruro de bencilo para iniciar la polimerización de la 2-metil-2-oxazolina debido al mejor desarrollo del carbocatión bencílico por la menor afinidad electrónica del átomo de yodo ${ }^{9,11}$. Posteriormente, la molécula de 2-metil-2-oxazolina ataca nucleofilicamente al carbocatión bencílico produciéndose el primer catión oxazolínico. Luego, este catión oxazolínico es atacado por nuevas moléculas de 2-metil-2-oxazolina produciéndose, así, la propagación de la polimerización. Para terminar la reacción se hace reaccionar el catión oxazolínico propagante con el hidróxido de potasio y se introduce al final de la cadena el grupo hidroxilo. De esta forma, se obtiene un macromonómero de 2-metil-2oxazolina de relativamente bajo peso molecular, el cual contiene un grupo vinilo al inicio de la cadena polimérica.

\section{Síntesis de copolímeros injertados mediante el método del macromonómero}

Fueron sintetizados nuevos copolímeros injertados mediante la polimerización por radicales libres de $\mathrm{N}$-isopropilacrilamida y los macromonómeros de 2-metil-2-oxazolina (MM-1 y MM-2) (esquema 2). La polimerización fue iniciada por el 2,2' -azoisobutironitrilo y llevada a cabo a $70^{\circ} \mathrm{C}$ en dimetilformamida. Se obtuvieron copolímeros injertados de tipo estadístico ya que se alimentó la mezcla de monómeros al inicio de la reacción. El rendimiento de la polimerización estuvo entre 48 y $94 \%$. En la tabla 2 se muestran los detalles experimentales y resultados obtenidos.

La estructura química de los copolímeros fue confirmada por sus espectros ${ }^{1} \mathrm{H}-\mathrm{RMN}$ (ejemplo CI-2 en figura 2). El contenido relativo de los comonómeros en el copolímero fue determinado a partir del análisis cuantitativo del espectro $1 \mathrm{H}-\mathrm{RMN}$ mediante la comparación de la integral de la señal "i" del metino $(\mathrm{CH})$ del grupo isopropilo del NIPAAm y de la señal "h" de los grupos metilenos $\left(\mathrm{NCH}_{2} \mathrm{CH}_{2}\right)$ del macromonómero. 

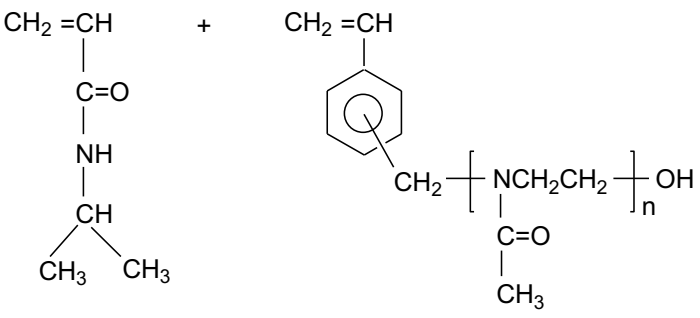

$$
\text { DMF, N } 2 \downarrow \begin{aligned}
& \text { AIBN } \\
& 70^{\circ} \mathrm{C}
\end{aligned}
$$

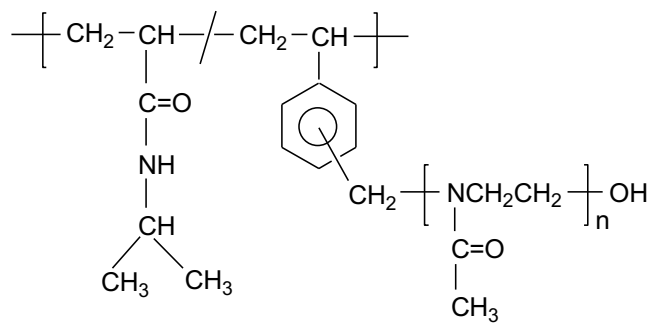

Esquema 2. Síntesis de los copolímeros injertados a partir de NIPAAm y macromonómero de 2-metil-2-oxazolina.

Se determinó que la relación molar NIPAAm/MM en el copolímero fue, en todos los casos, mayor a la relación molar inicial de estos comonómeros y fue aún mayor en el caso de MM-2 (tabla 2). Esto indica que el NIPAAm se incorporó en mayor cantidad que los macromonómeros (MM-1 o MM-2) en el copolímero. Este resultado era esperado ya que el monómero NIPAAm, por ser una molécula de bajo peso molecular, tiene una mayor velocidad de difusión que el macromonómero, que es un polímero, y por esta razón el NIPAAm puede reaccionar más fácilmente con la especie propagante radicalaria y se incorporaría en mayor cantidad en el copolímero. En la literatura no existen valores para las razones de reactividad de este nuevo sistema de copolimerización pero en un sistema similar que consiste de estireno (M1) y N,N-dietilacrilamida $\left(\mathrm{M}_{2}\right)$, los valores de reactividad son $\mathrm{r} 1$ $=1,23 \mathrm{y} \mathrm{r}_{2}=0,39$, respectivamente ${ }^{18,19}$. Llevando estos datos al presente caso, eso indicaría que el grupo estirilo del macromonómero tendría una mayor reactividad que el NIPAAm y así, por este factor, se favorecería una mayor incorporación de MM en el copolímero en el inicio de la polimerización. Sin embargo, debido a la baja difusibilidad del MM, por su peso molecular relativamente alto en comparación al NIPAAm, al final se incorporaría de preferencia el NIPAA en el copolímero, debido a su bajo peso molecular y por consiguiente mayor difusibilidad en el medio reaccionante. Este último factor es lo que al final predomina en la copolimerización. 
El peso molecular de los copolímeros injertados y macromonómeros no fue determinada por cromatografía de permeación en gel (GPC) debido a que, generalmente, con polímeros anfífilicos, como es este caso, ya fue demostrado en la literatura que se producen resultados inesperados, por ejemplo, se obtiene un muy bajo peso molecular y una muy ancha distribución del peso molecular ${ }^{18,19}$. Esto ocurre, probablemente, debido a que, por su carácter anfifílico (conteniendo simultáneamente dentro de su estructura partes hidrofóbicas e hidrofílicas), estos polímeros tienden a adherirse a las columnas cromatográficas y estos polímeros también pueden formar micelas o agregados moleculares en la mezcla de solventes del GPC (ejemplo: dimetilformamida /agua/ $\mathrm{LiCl})^{18,20}$.

Tabla 2. Síntesis de los copolímeros injertados. Condiciones de reacción y resultados.

\begin{tabular}{|c|c|c|c|c|c|c|c|}
\hline $\mathrm{CI}^{\mathrm{a}}$ & $\mathrm{MM}^{\mathrm{b}}$ & $\begin{array}{l}\text { NIPAAm }^{\mathrm{c}} \\
(\mathrm{mmol})\end{array}$ & $\begin{array}{c}\mathrm{MM}^{\mathrm{d}} \\
(\mathrm{mmol})\end{array}$ & $\begin{array}{l}\text { AIBN }^{\mathrm{e}} \\
(\mathrm{mmol})\end{array}$ & $\mathrm{R} 1^{\mathrm{f}}$ & $\mathrm{R} 2^{\mathrm{g}}$ & $\begin{array}{l}\operatorname{LCST}^{\mathrm{h}} \\
\left({ }^{\circ} \mathrm{C}\right)\end{array}$ \\
\hline CI-1 & MM-1 & 2,78 & 0,19 & 0,07 & 15 & 17 & 37,5 \\
\hline CI-2 & MM-1 & 11,16 & 0,37 & 0,29 & 30 & 35 & 35 \\
\hline CI-3 & MM-1 & 11,16 & 0,19 & 0,29 & 61 & 70 & 34 \\
\hline CI-4 & MM-1 & 15,91 & 0,19 & 0,40 & 84 & 100 & 33 \\
\hline CI-5 & MM-2 & 0,37 & 4,42 & 0,12 & 12 & 16 & 38 \\
\hline CI-6 & MM-2 & 8,59 & 0,29 & 0,22 & 30 & 50 & 35 \\
\hline CI-7 & MM-2 & 11,45 & 0,19 & 0,29 & 60 & 87 & 34 \\
\hline CI-8 & MM-2 & 11,45 & 0,10 & 0,29 & 120 & i) & 33,5 \\
\hline
\end{tabular}

\footnotetext{
a) Denominación del copolímero injertado, b) Macromonómero, c) $\mathrm{N}$-isopropilacrilamida, d) Macromonómero $\mathrm{MM},{ }^{\text {e) }}$ 2,2' -Azoisobutironitrilo, ${ }^{\text {f) }} \mathrm{R} 1$ = Relación molar de NIPAAm/MM en la alimentación al reactor, $\left.{ }^{g}\right)$ R2 = Relación molar final de NIPAAm/MM en el copolímero injertado fue determinada mediante el análisis cuantitativo del espectro ${ }^{1} \mathrm{H}-\mathrm{RMN}$, h) Temperatura de transición conformacional (LCST) del copolímero injertado, i) No fue determinado, j) Los rendimientos de la síntesis de copolímeros injertados fueron de 67, 82, 52, 48, 62, 94, 78, у 69 para CI-1, CI-2, hasta CI-8, respectivamente.
}

\section{Determinación de la temperatura de transición de los copolímeros injertados}

Los copolímeros injertados también fueron caracterizados por su temperatura de transición conformacional (LCST), la cual fue determinada por el método turbidimétrico (espectrómetro UV/Vis) y espectros ${ }^{1} \mathrm{H}-\mathrm{RMN}$. El método turbidimétrico consistió en la medición del porcentaje de transmitancia de soluciones acuosas de copolímero injertado versus la temperatura. El polímero con una sensibilidad térmica precipita a una determinada temperatura y entonces el porcentaje de transmitancia disminuye. 
En estas mediciones se usó como elemento de comparación el poliNIPAAm comercial (Aldrich, peso molecular $\mathrm{Mn}=20000$ ). El poliNIPAAm "puro" tuvo una transición conformacional aproximadamente a los $31,5^{\circ} \mathrm{C}\left(\right.$ Lit. $\left.32^{\circ} \mathrm{C} 4\right)$, en la que cambió drásticamente su característica pasando de ser hidrofílico a hidrofóbico y ocurrió una repentina dispersión del polímero en el agua de tal manera que se interrumpió el paso de la luz y la transmitancia cayó hasta un valor de cero $^{4,7}$ (figuras 3 y 4 ).

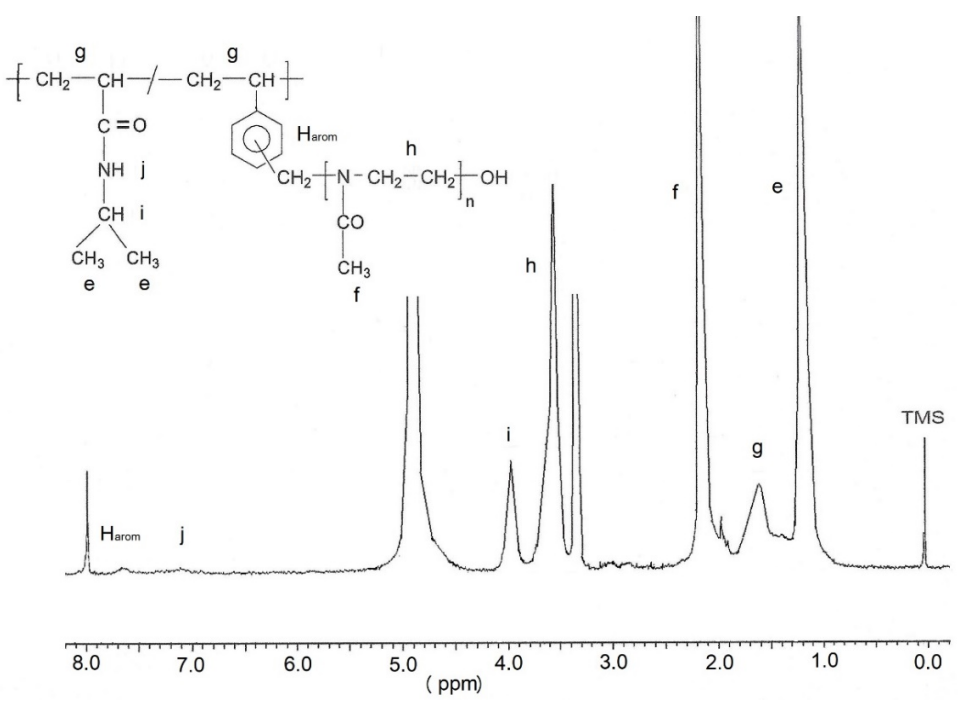

Figura 2. Espectro ${ }^{1} \mathrm{H}-\mathrm{RMN}$ del copolímero injertado CI-2 en metanol deuterado a $25{ }^{\circ} \mathrm{C}$. Las señales del espectro han sido asignadas a la estructura del polímero. Las señales en 3, y 4,9 ppm corresponden a las señales del metanol deuterado.

Respecto a los copolímeros injertados, se observó que la inclusión de los segmentos hidrofílicos de los macromonómeros incrementaron el valor de la LCST y este incremento fue mayor a mayor cantidad de macromonómero y también se determinó que al aumentar el contenido de MM en el copolímero injertado disminuyó la intensidad de la transición conformacional (los valores de transmitancia no llegaron a valores cercanos a cero) y esta disminución de la intensidad de la transición conformacional fue más acentuada cuando el peso molecular del macromonómero fue mayor (comparar, por ejemplo, CI-5 (MM2) vs CI-1 (MM-1)). Estos resultados experimentales se podrían explicar de la siguiente manera (Zschoche y Rueda ${ }^{15,16,18}$ ): los segmentos hidrofílicos de polimetiloxazolina del macromonómero producen una mayor hidrofilicidad que los segmentos de poliNIPAAm en la cadena principal y, por consiguiente, un corrimiento de la LCST hacia temperaturas más altas. A partir del LCST, los copolímeros con un contenido de poliMeOxa relativamente alto podrían formar micelas o agregados moleculares del tipo "core-shell”, las cuales contendrían un núcleo hidrofóbico de poliNIPA colapsado y en el exterior de este núcleo estarían las 
cadenas laterales hidrofílicas de poliMeOxa, solvatadas por las moléculas de agua ${ }^{16,18}$ (esquema 3). Este fenómeno estabilizaría las micelas y parcialmente impediría que ocurra el colapso intermolecular entre las diferentes partículas poliméricas.

En el caso de los macromonómeros MM-1 y MM-2, también se les sometió a las mismas mediciones que los copolímeros injertados, pero no se observó ninguna dispersión del polímero ya que estos polímeros no son sensibles a la temperatura (figuras 3 y 4).

En el caso de los copolímeros injertados CI-1 y CI-5, también se determinó la temperatura de transición conformacional (LCST) mediante los espectros ${ }^{1} \mathrm{H}-\mathrm{RMN}$. Se obtuvo los espectros ${ }^{1} \mathrm{H}-\mathrm{RMN}$ de cada copolímero injertado a temperaturas crecientes y se pudo observar que, a una determinada temperatura, ocurrió un decrecimiento de la intensidad de las señales que corresponden a las unidades de NIPAAm contenidas en la cadena principal del copolímero injertado (figura 5). Por ejemplo, la señal ubicada entre 1,0 y 1,3 ppm perteneciente a los dos grupos metilo del NIPAAm no sufrió cambios desde los 25 hasta los $35^{\circ} \mathrm{C}$, aproximadamente. Sin embargo, a partir de esta última temperatura ocurrió una disminución de la intensidad de esta señal, la cual decreció paulatinamente hasta aproximadamente los $60{ }^{\circ} \mathrm{C}$ y se estabilizó. Al ocurrir el LCST, los segmentos de poliNIPA contenidos en la cadena principal se aglomeran, colapsan, y son solo parcialmente solvatados por las moléculas de agua, entonces disminuye fuertemente su movilidad, se comportan parcialmente como sólidos, tienen un alto tiempo de relajación en el RMN y entonces son detectados con menos intensidad en el espectro 1H-RMN. La señal del metilo ("e") no desaparece totalmente, probablemente debido a que las cadenas laterales de poliMeOXA, fuertemente hidrofílicas, permiten, por su cercanía a las cadenas de poliNIPAAm, una solvatación parcial de estas por las moléculas de agua deuteradas y, por consiguiente, existe todavía una movilidad parcial.

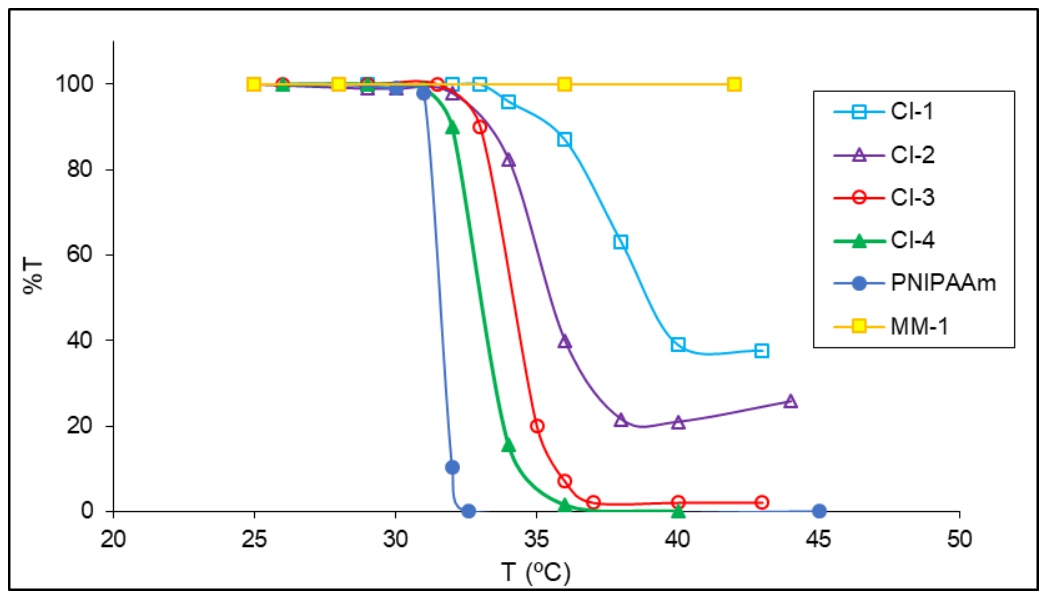

Figura 3. Determinación del LCST de los copolímeros injertados CI-1 - CI-4 mediante el método turbidimétrico. Los valores de LCST fueron 37,5, 35, 34, 33 y 31,5 para CI-1, CI-2, CI-3, CI-4, y PoliNIPAAm, respectivamente. (Gráfica del porcentaje de transmisión $(\% \mathrm{~T})$ vs temperatura $\left({ }^{\circ} \mathrm{C}\right), 500$ $\mathrm{nm}, 1 \%$ en peso de solución polimérica). PoliNIPAAm $(\mathrm{Mn}=20000)$. 
Por el contrario, las señales correspondientes a las cadenas de poli(2-metil-2-oxazolina) si son detectadas hasta, relativamente, altas temperaturas como, por ejemplo, $75^{\circ} \mathrm{C}$ (figura 5 , señales "f" y "h") debido a que éstas, por su hidrofilicidad, si son bien solvatadas por las moléculas de agua, aun a temperaturas encima de $\operatorname{los} 35^{\circ} \mathrm{C}$ y entonces tienen movilidad y aparecen en el espectro RMN.

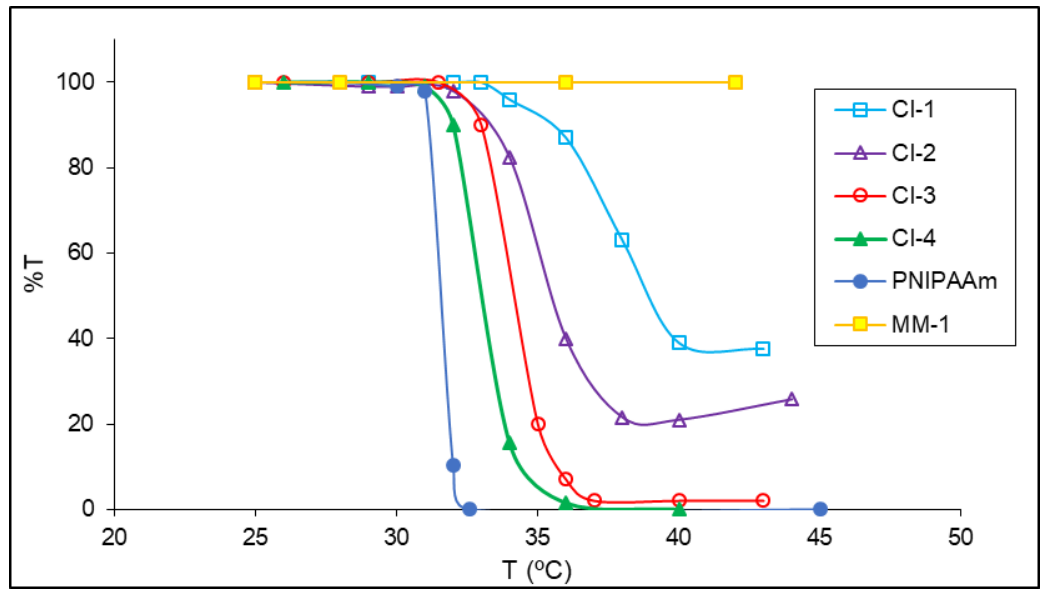

Figura 4. Determinación del LCST de los copolímeros injertados CI-5, CI-6, CI-7, y CI-8 mediante el método turbidimétrico. Los valores de LCST fueron 38, 35, 34, 33,5 y 31,5 para CI-5, CI-6, CI-7, CI8 , y PoliNIPAAm, respectivamente (gráfica del porcentaje de transmisión $(\% \mathrm{~T})$ vs temperatura $\left({ }^{\circ} \mathrm{C}\right)$, $500 \mathrm{~nm}, 1 \%$ en peso de solución polimérica). PoliNIPAAm $(\mathrm{Mn}=20000)$.
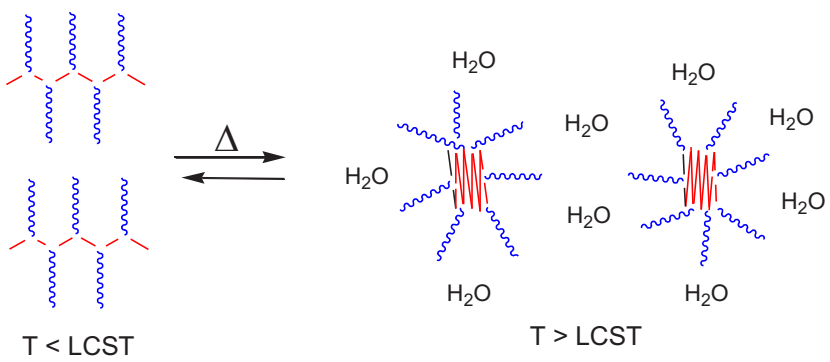

Esquema 3. Modificación conformacional de los copolímeros injertados que contienen una cantidad relativamente alta de cadenas laterales de poliMeOxa (CI-1, CI-2, CI-5, CI-6) respecto a la cadena principal de NIPAAm.

Este comportamiento obtenido en el espectro RMN corrobora los resultados encontrados con el método turbidimétrico y se pueden explicar, cómo fue mencionado líneas arriba, por la formación de micelas o agregados moleculares a temperaturas mayores a $35^{\circ} \mathrm{C}$ (esquema 3 ). 

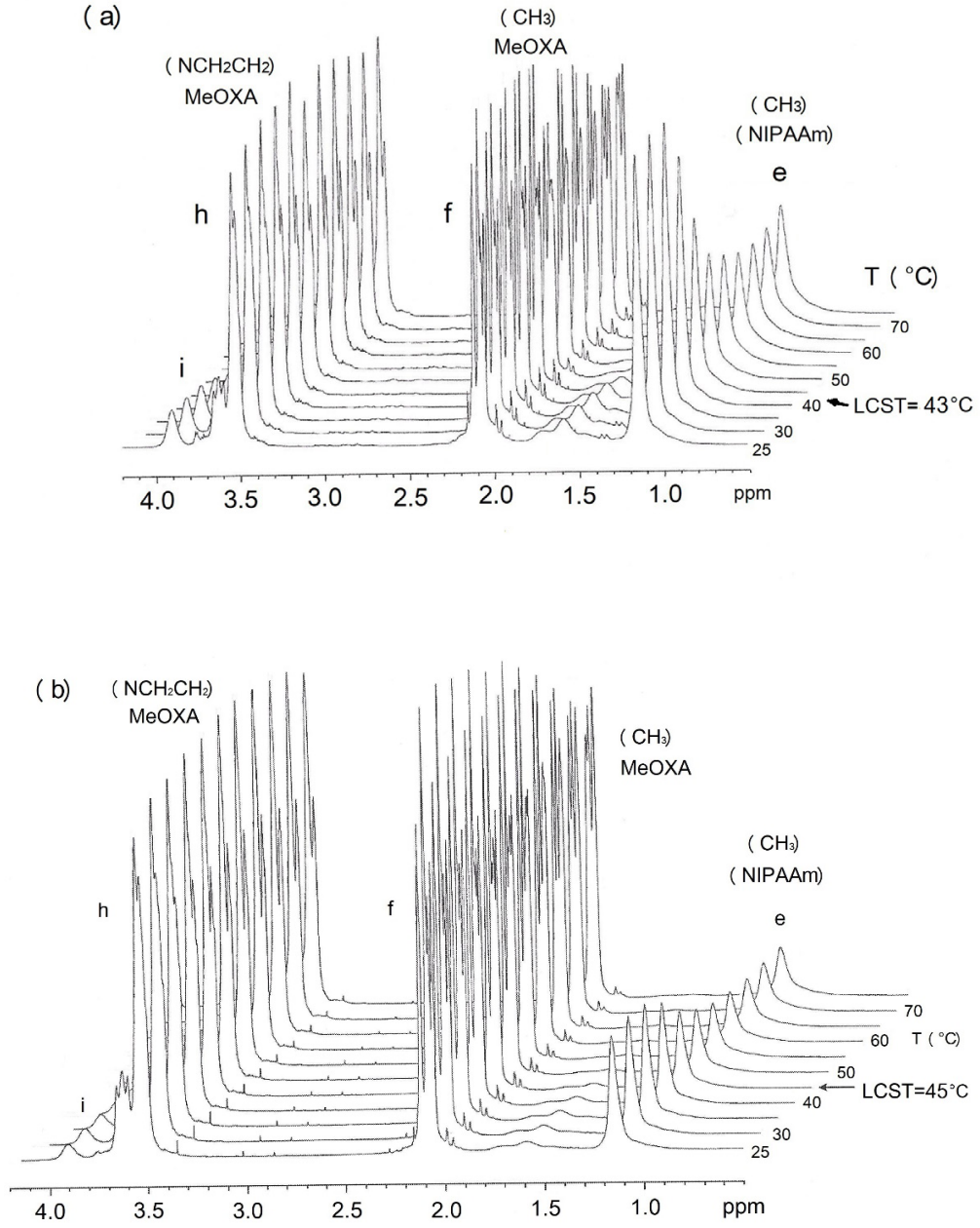

Figura 5. Espectro ${ }^{1} \mathrm{H}-\mathrm{RMN}$ del copolímero injertado CI-2 en metanol deuterado a $25{ }^{\circ} \mathrm{C}$. Las señales del espectro han sido asignadas a la estructura del polímero. Las señales en 3, y 4,9 ppm corresponden a las señales del metanol deuterado.

La corona hidrofílica de poliMeOxa, solvatada por las moléculas de agua, estabiliza y separa unas micelas de otras y esto impide, por lo menos parcialmente, un colapso intermolecular de los polímeros, pero sí ocurre el colapso intramolecular.

Para hallar el valor de la temperatura de transición conformacional se graficó la intensidad de la señal del grupo metilo del NIPAAm versus la temperatura y se obtuvo una curva y se tomó el LCST como el punto de inflexión de la misma (Figura 6, ej. CI-1) Se obtuvo los valores aproximados de LCST de $43{ }^{\circ} \mathrm{C}$ y $45^{\circ} \mathrm{C}$ para CI-1 y CI-5, respectivamente. Estos valores son 
mayores a los obtenidos vía el método turbidimétrico, que son 37,5 y $38^{\circ} \mathrm{C}$ para CI-1 y CI5 , respectivamente. Esta diferencia puede deberse a que, en el método RMN, la medición se hizo más rápido que en el método turbidimétrico y entonces el polímero no consiguió llegar a su equilibrio total en cada temperatura.

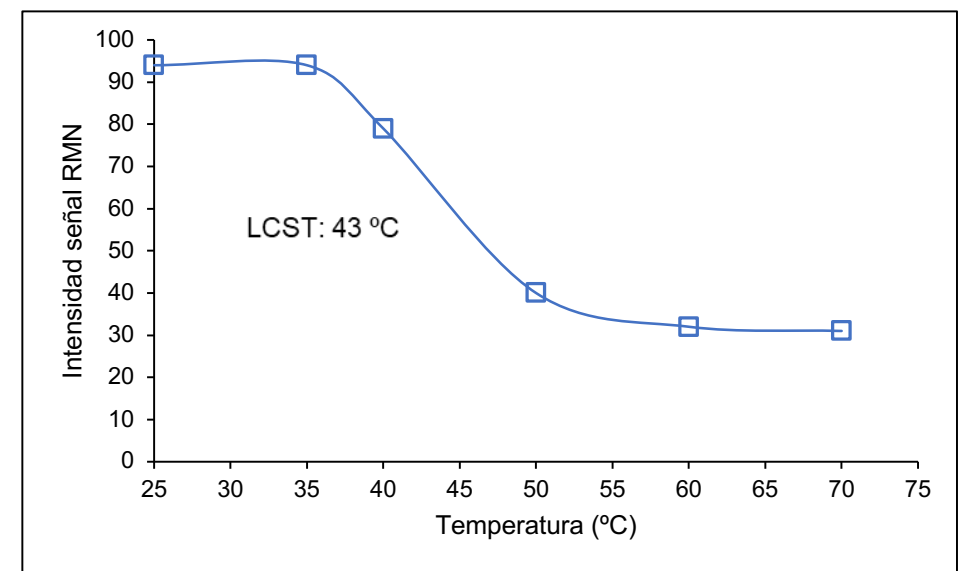

Figura 6. Determinación del LCST del copolímero CI-1 mediante 1H-RMN. Gráfica de la intensidad de la señal RMN del grupo metilo del NIPAAm en función de la temperatura.

\section{CONCLUSIONES}

Fueron sintetizados nuevos copolímeros injertados mediante la polimerización por radicales libres de N-isopropilacrilamida (NIPAAm) y macromonómeros de 2-metil-2oxazolina (MM) iniciada por el $2,2^{\prime}$-azobisisobutironitrilo a $70{ }^{\circ} \mathrm{C}$ en dimetilformamida. Los macromonómeros fueron sintetizados mediante la polimerización catiónica por apertura de anillo de 2-metil-2-oxazolina iniciada por el clorometilestireno en presencia de ioduro de sodio a $78{ }^{\circ} \mathrm{C}$ en acetonitrilo. Los copolímeros injertados mostraron una transición conformacional (LCST) a una determinada temperatura, la cual fue en función del grado de polimerización del macromonómero y de la variación de la razón molar de NIPAAm/ macromonómero dentro del copolímero injertado. La temperatura de transición LCST se incrementó con la disminución de la razón molar NIPAAm/MM en el copolímero y la transición conformacional perdió intensidad al aumentar el porcentaje molar de MM en el copolímero injertado y al aumentar el grado de polimerización del macromonómero. A partir de la temperatura LCST, los copolímeros injertados con un relativamente alto contenido de MM podrían formar micelas o agregados moleculares tipo "core-shell” en solución acuosa que estarían formados por un núcleo hidrofóbico de poliNIPAAm colapsado y una corona formada de cadenas hidrofílicas de polimetiloxazolina solvatadas por las moléculas de agua que impedirían parcialmente un colapso intermolecular de estos agregados. 


\section{AGRADECIMIENTOS}

Los autores desean agradecer a la Dirección de Gestión de la Investigación (DGI) de la Pontificia Universidad Católica del Perú por el apoyo financiero para esta investigación y al Dr. Hartmut Komber, del Instituto Leibniz de Investigaciones en Polímeros de Dresden (IPF), Alemania por los análisis de resonancia magnética nuclear (RMN).

\section{REFERENCIAS BIBLIOGRÁFICAS}

1. Koetting MC, Peters JT, Steichen SD, Peppas NA. Stimulus-responsive hydrogels: Theory, modern advances, and applications. Mater Sci Eng R Rep. 2015; 93:1-49.

2. Aguilar M, San Roman J. Smart polymers and their applications. Second edition. London, England: Elservier; 2019.

3. Cohen SA, Huck TS, Genzer J, Muller M, Stamm M. Emerging applications of stimuliresponsive polymer materials. Nat Mater. 2010; 9:101-113.

4. Schild HG. Poly(N-isopropylacrylamide): experiment, theory and application. Prog Polym Sci. 1992; 17: 163-249.

5. Rueda JC, Zschoche S, Komber H, Krahl F, Arndt KF, Voit B, New Thermo-Sensitive Graft Copolymers Based on a Poly(N-isopropylacrylamide) Backbone and Functional Polyoxazoline Grafts with Random and Diblock Structure Macromol Chem Phys. 2010; 211: 706-711.

6. Krause AT, Zschoche S, Rohn M, Hempel C, Richter A, Appelhans D, et al. Swelling behavior of bisensitive interpenetrating polymer networks for microfluidic applications. Soft Matter. 2016; 12: 5529-5536.

7. Schmaljohann D. Thermo- and $\mathrm{pH}$-responsive polymers in drug delivery. Adv Drug Deliv Rev. 2006;58(15):1655-70.

8. Pizzi D, Humphries J, Morro J, Fletcher NL, Bell CA, Thurecht KJ, et al. Poly(2oxazoline) macromonomers as building blocks for functional and biocompatible polymer architectures. Eur Polym J. 2019; 121:109258. doi: 10.1016/j.eurpolymj.2019.109258.

9. Rueda JC, Santillán F, Komber H, Voit B. Synthesis and Characterization of Stiff, Self-Crosslinked Thermoresponsive DMAA Hydrogels. Polymers. 2020: 12:1401. doi: 10.3390/polym 12061401

10. Rueda JC, Campos E, Komber H, Zschoche S, Haussler L, Voit B. Synthesis and characterization of new $\mathrm{pH}$ - and thermo-responsive hydrogels based on N-isopropylacrylamide and 2-oxazolines. Des. Monomers Polym. 2014: 17: 208-216.

11. Rueda JC, Suárez C, Komber H, Zschoche S, Voit B. Synthesis and characterization of $\mathrm{pH}$ - and thermo-responsive hydrogels based on poly(2-cyclopropyl-2-oxazoline) macromonomer, sodium acrylate, and acrylamide. Polymer Bull. 2020; 77: 5553-5565.

12. Lava K, Verbraeken B, Hoogenboom R. Poly(2-oxazoline)s and click chemistry: a versatile toolbox toward multi-functional polymers. Eur Polym J. 2015; 65: 98-111.

13. Jana S, Uchman M. Poly(2-oxazoline)-based Stimulus-Responsive (Co)polymers: An Overview of their Design, Solution Properties, Surface-chemistries and Applications. Prog Polym Sci. 2020; 106: 101252. doi: 10.1016/j.progpolymsci.2020.101252. 
14. Verbraeken B, Monnery B, Lava K, Hoogenboom R. The chemistry of poly(2-oxazoline) s. Eur Polym J. 2017; 88: 451-469.

15. Zschoche S, Rueda JC, Binner M, Komber H, Janke A, Arndt KF, et al. Reversibly Switchable pH- and Thermoresponsive Core-Shell Nanogels Based on Poly(NiPAAm)graft-poly(2-carboxyethyl-2-oxazoline)s. Macromol Chem Phys. 2012; 213: 215-226.

16. Zschoche S, Rueda JC, Komber H, Volodymyr B, Arndt KF, Voit B. Thermo-Responsive Nanogels Based on Poly[NIPAAm-graft-(2-alkyl-2-oxazoline)]s Crosslinked in the Micellar State. Macromol Chem Phys. 2010; 211: 1035-1042.

17. Witte H, Seeliger W. Cyclische Imidsäureester aus Nitrilen und Aminoalkoholen. Eur J Org Chem. 1974; 6: 996-1009.

18. Rueda JC, Zschoche S, Komber H, Schmaljohann D, Voit B. Synthesis and Characterization of Thermoresponsive Graft Copolymers of NIPAAm and 2-Alkyl-2oxazolines by the "Grafting from" Method. Macromolecules. 2005; 38: 7330-7736.

19. Greenley RZ. Free radical copolymerization reactivity ratios. In: Brandrup J, Immergut EH. Polymer Handbook. Third edition. New York, USA: J. Wiley \& Sons; 1989.

20. Meijs GF, Rizzardo E. Reactivity of macromonomers in free radical polymerization. J Polym Sci. Part C: Polym Lett. 1990; 30: 305-377. 